\title{
GMR
}

\section{Methylation profile of SNRPN gene and its correlation with weight and chronological age}

\author{
N.V. Carobin, F.V.M. Rubatino, M.L. Freitas, V.T. de Oliveira, R.X. Pietra, \\ A.A. Bosco and F.S. Jehee \\ Instituto de Ensino e Pesquisa da Santa Casa de Belo Horizonte, \\ Belo Horizonte, MG, Brasil \\ Corresponding author: N.V. Carobin \\ E-mail: natyvirca@gmail.com \\ Genet. Mol. Res. 14 (4): 13791-13798 (2015) \\ Received April 23, 2015 \\ Accepted July 24, 2015 \\ Published October 28, 2015 \\ DOI http://dx.doi.org/10.4238/2015.October.28.41
}

ABSTRACT. Genomic imprinting is an important epigenetic phenomenon, wherein genes or gene clusters are marked by DNA methylation during gametogenesis. This plays a major role in several functions of normal cells, including cell differentiation, $X$ chromosome inactivation, and the maintenance of chromatin structure, in mammalian development. The aim of this study was to investigate the possible differences in SNRPN gene methylation profiles in non-obese and obese individuals, and in children and adults. Our results did not reveal any statistical correlations between the DNA methylation profiles of the SNRPN gene in children or adult obese and non-obese groups. However, a comparison of the methylation levels with the chronological age revealed statistically significant differences between the means of methylation in adults and children (46.20 \pm 5.88 and $39.40 \pm 2.87$, respectively; $P<0.001$ ). Pearson's correlation analysis indicated a positive association between the level of DNA methylation and the chronological age $\left(R^{2}=0.326 ; P<0.001\right)$. Therefore, we concluded that the methylation profile of the SNRPN promoter (in blood) is not a useful biomarker for determining the predisposition of an individual to obesity. Additionally, we have confirmed that SNRPN methylation increases with 
age, which raises further questions regarding the role of SNRPN expression during the aging process.

Key words: Methylation level; SNRPN; Prader-Willi syndrome; Obesity; Aging process

\section{INTRODUCTION}

Methylation and genomic imprinting are the most widely studied phenomena in the context of CpG dinucleotides in promoter regions (Christensen et al., 2009) Essential functions of normal mammalian development, such as cell differentiation, X-chromosome inactivation, and chromatin structure maintenance, are determined by DNA methylation (Weinstein et al., 2010). Genomic imprinting occurs when genes or gene clusters are marked by DNA methylation during gametogenesis, leading to the silencing of specific alleles; this results in monoallelic parent-oforigin-specific expression in the offspring (Reik and Walter, 2001). Therefore, the study of genomic imprinting is of particular importance in mammals and might provide novel insights into gene expression and human development.

Genome-wide studies of methylation in normal human tissues have shown dynamic epigenetic changes in the DNA methylation profiles, caused by environmental factors and aging processes (Schilling and Rehli, 2007; Rakyan et al., 2008). In fact, recent works have demonstrated an overall trend of increased methylation associated with older age in normal human tissues (Shen et al., 2005; Kwabi-Addo et al., 2007).

Some syndromes leading to abnormal growth and severe obesity, including the BeckwithWiedemann, Silver-Russell, and Prader-Willi (PWS) syndromes, are associated with defective genomic imprinting (Souren et al., 2011). The lack of expression of SNRPN, as well as other genes, such as NDN1 and MAGEL2, in the 15q11.2-q13 region, results in the onset of hyperphagia, loss of satiety, and obesity in PWS (Bischof et al., 2007).

Previous studies have already reported on the relationship between the increase in DNA methylation and body mass index (BMI); however, little is known about the methylation status of SNRPN in non-syndromic obese subjects, and its effect on the onset and development of obesity from childhood to adulthood (Wang et al., 2010; Crujeiras et al., 2013; Horvath et al., 2014).

This study, therefore, aims to quantify the methylation percentage of the SNRPN locus, and investigate its correlation with BMI and age in obese and non-obese children and adult subjects.

\section{MATERIAL AND METHODS}

\section{Samples and ethics statement}

Peripheral blood samples were collected in ethylene diamine tetraacetic acid tubes from fifty non-syndromic subjects. Individuals were divided into various groups as follows: group 1, fifteen obese adults (seven male and eight female) (BMI $\geq 40.0$ ); group 2, fifteen non-obese adults (seven male and eight female) (BMI 18.6-29.9); group 3, ten obese children (five male and five female) (BMI $\geq 95$ th); and group 4, ten non-obese children (five male and five female) (BMI $\geq 5$ th < 85th) (WHO, 2000; Mei et al., 2002). All participants and/or their parents were asked to sign informed consent forms, drawn up according to the guidelines of the local Ethics Committee. 


\section{DNA extraction and bisulfite treatment}

Genomic DNA was extracted from the peripheral blood samples using the Gentra Puregene Blood Kit (Qiagen Germantown, MD, USA), according to the manufacturer instructions. These samples were then subjected to bisulfite treatment using the EpiTect Plus DNA Bissulfite Kit (Qiagen, Hilden, NRW, German), as per the instructions of the manufacturer.

\section{Primer design and sequences}

Primers sequences for the amplification of the SNRPN (NG_012958.1) gene were designed based on the methodologies of Rubatino et al., 2015. In summary, the designed forward primer established a perfect match with the unmethylated template, while the reverse primer exhibited one mismatch with the methylated template. Figure 1 indicates the forward and reverse primers sequences at their binding sites.

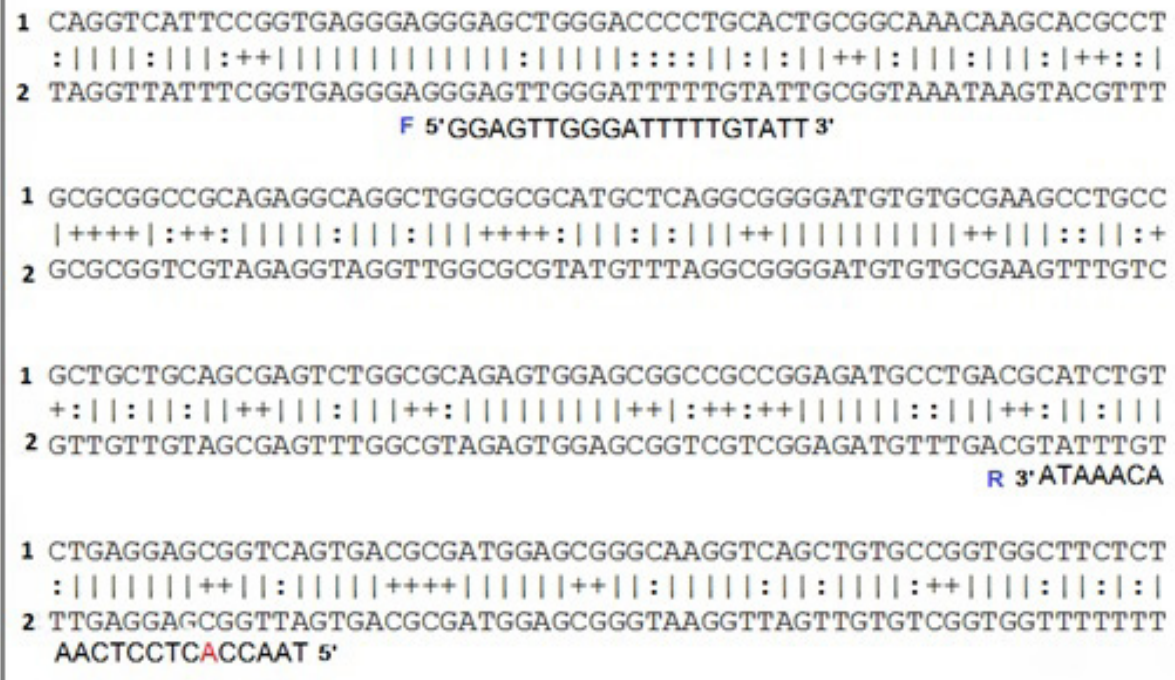

Figure 1. Forward (F-5'-GGAGTTGGGATTTTTGTATT-3') and reverse (R-3'-ATAAACAAACTCCTCACCAAT-5') primer sequences at their binding sites in exon 1 of SNRPN. The mismatch is highlighted in red in the reverse primer sequence. Line one represents the original DNA sequence; line two shows the DNA sequence after bisulfite treatment. "++" refers to CpG sites and ":" refers to C modified to T after bisulfite treatment. SNRPN (NG_012958.1).

\section{Polymerase chain reaction (PCR) conditions and controls}

All reactions were carried out in a final volume of $10 \mu \mathrm{L}$, containing 2 ng bisulfite-converted DNA, $0.175 \mu \mathrm{M}$ of each SNRPN primer, 1X MeltDoctor Master Mix (Applied Biosystems, Foster City, CA, USA). The PCR cycle conditions were set as follows: an initial denaturation at $95^{\circ} \mathrm{C}$ for $10 \mathrm{~min}$, and 45 cycles of $95^{\circ} \mathrm{C}$ for $25 \mathrm{~s}, 52.5^{\circ} \mathrm{C}$ for $20 \mathrm{~s}$, and $67^{\circ} \mathrm{C}$ for $40 \mathrm{~s}$. The samples were melted at the temperature ramping and fluorescence acquisition setting recommended by the manufacturer, immediately following the complete PCR cycle. All reactions were performed in duplicate on an ABI 7500 Fast RealTime PCR System (Applied Biosystems), and analyzed using High Resolution Melt software (HRM). 


\section{Determination of the standard curve and methylation samples}

The standard curve was constructed from samples with known differences in the levels of methylation $(0,25,50,75$, and $100 \%)$. Bisulfite-converted DNA extracted from subjects previously diagnosed with Prader-Willi syndrome (herein considered as being $100 \%$ methylated) and Angelman (herein considered as being $0 \%$ methylated) syndromes were utilized as controls.

The differences calculated using the $0 \%$ methylated sample as the baseline were plotted on the $y$-axis of the fluorescence plot (DP); the values of the known methylation percentages were plotted on the $\mathrm{x}$-axis. The methylation levels were determined from the subjects by applying mean values of the differences in the fluorescence plot to the standard curve equation.

\section{Statistical analysis}

The methylation percentages calculated from the subjects were inputted into a SPSS (v.20; IBM, Armonk, NY, USA) data bank. A Kolmogorov-Smirnov adherence test was conducted before initiating the analysis. The sample size was determined based on an independent sample, $\alpha=5 \%$ and $\beta$ error $=20 \%$ (Miot, 2011). The minimal difference that was accepted between the groups was $5 \%$. The means were compared using a $t$-test. The relationship between methylation and BMI, and methylation and age, was analyzed using the Pearson's test, maintaining a confidence interval of $95 \%$ for both groups.

\section{RESULTS}

The PCR and melting procedures were performed as previously described for all subjects, accompanied by a standard curve, as shown in Figure 2.

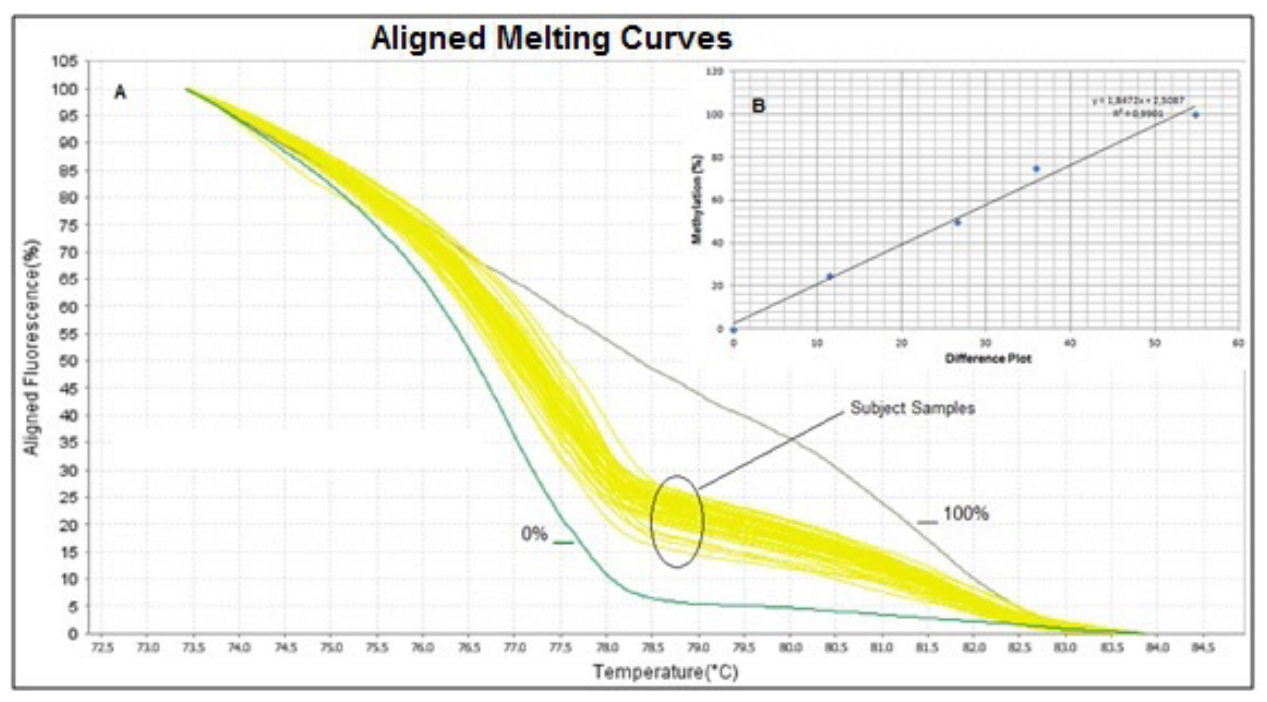

Figure 2. Aligned melting curves for polymerase chain reaction (PCR) of the SNRPN promoter region. (A) From left to right, aligned melting curves $0 \%$ methylated samples, sample subjects, and 100\% methylated samples; (B) Standard curve. 
Table 1 summarizes the means and standard deviations of chronological age and body mass indices (BMI) of the subjects and controls, and the methylation levels of SNRPN; these values were obtained from each subject, and comparisons and possible associations between the subjects were identified.

Table 1. Mean age, body mass index (BMI), and methylation in obese and non-obese adults and children.

\begin{tabular}{lccr}
\hline Adult & Obese $(\mathrm{N}=15)$ & Non-obese $(\mathrm{N}=15)$ & $\mathrm{P}$ value \\
\hline Age (mean $\pm \mathrm{SD})$ & $40.0 \pm 10.3$ & $35 \pm 12,8$ & 0.238 \\
BMI (mean $\pm \mathrm{SD})$ & $42.6 \pm 7.9$ & $22,0 \pm 1,6$ & $<0.001$ \\
Methylation (mean $\pm \mathrm{SD})$ & $45.7 \pm 5.3$ & $46,6 \pm 6,4$ & 0.672 \\
Children & Obese $(\mathrm{N}=10)$ & Non-obese $(\mathrm{N}=10)$ & \\
Age (mean $\pm \mathrm{SD})$ & $12.0 \pm 2.7$ & $10.0 \pm 2.6$ & 0.113 \\
BMl (mean $\pm \mathrm{SD})^{*}$ & $31.0 \pm 7.4$ & $16.3 \pm 2.7$ & $<0.001$ \\
Methylation (mean $\pm \mathrm{SD})$ & $39.7 \pm 2.7$ & $39.1 \pm 3.1$ & 0.653 \\
\hline
\end{tabular}

*According to Mei (2002), children are classified into the healthy weight or non-obese group when the weight is between the 5th and 85th percentile and in the obese category when the weight exceeds the 95th percentile. SD = standard deviation.

The results of this study revealed that the methylation levels of the SNRPN promoter did not differ statistically between obese and non-obese individuals, in the adult or the child groups ( $P$ $=0.672$ and 0.653 , respectively). This suggests the lack of association between methylation levels of SNRPN and BMI in these subjects.

The subjects were rearranged into two new groups with the purpose of studying a possible association between SNRPN methylation levels and the chronological age: adults ( $\geq 18 \leq 66$ years) and children ( $\geq 6 \leq 15$ years). The mean age of the subjects in the two groups was 37.5 (SD \pm 11.75 ; adults) and 11 (SD \pm 5.84 ; children) years. The mean methylation percentage of SNRPN was $46.20 \%$ $(\mathrm{SD} \pm 5.88)$ in adults and $39.40 \%(\mathrm{SD} \pm 2.87)$ in children. Our results revealed that the methylation levels of SNRPN was significantly different between the two groups $(P<0.001)$. Pearson's correlation analysis comparing the SNRPN methylation with the chronological age indicated a positive correlation between the two factors $\left(R^{2}=0.326 ; P<0.001\right)$. This indicated that the higher methylation level of $S N R P N$ is correlated with an increase in the chronological age (Figure 3 ).

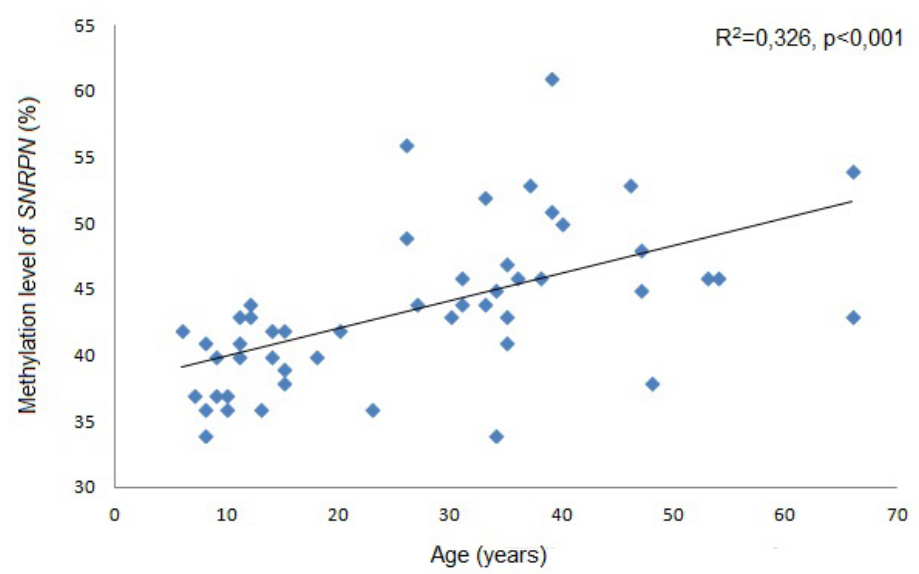

Figure 3. Pearson's correlation. Comparison between the methylation levels of SNRPN (\%) and the chronological age (years) in all test individuals. 


\section{DISCUSSION}

Methylation of the $\mathrm{CpG}$ islands in the gene promoter regions is responsible for controlling approximately $60 \%$ of the transcription in humans (Suzuki and Bird, 2008). This mechanism assists in gene silencing, and plays an important role in gene expression, embryonic growth, differentiation, and human development (Plasschaert and Bartolomei, 2014). The PWS region at 15q11.2-q13 is known to be strongly related to the onset of obesity; however, little is known of the relationship between gene methylation in this region and non-syndromic obesity.

SNRPN methylation levels in obese children and adults were $39.7 \pm 2.7$ and $45.7 \pm 5.3$, respectively, and non-obese children and adults were $39.1 \pm 3.1$ and $46.6 \pm 6.4$, respectively. Based on these results, no significance differences were observed between the obese and nonobese groups. Kim et al. (2012) used a different approach to measure the SNRPN methylation in a group of 80 subjects with early-onset morbid obesity; although the results of their study showed no statistically significant differences, the mean methylation in exon 1 of $\operatorname{SNPRN}(0.54 \pm 00.6)$ did not differ from the mean methylation level seen in the control group of four subjects $(0.55 \pm 00.7)$. These results also suggest that the DNA methylation profile of SNRPN (in blood) does not correlate to the BMI (Kim et al., 2012).

On the other hand, Crujeiras et al. (2013) demonstrated a relationship between weight and epigenetic alterations by measuring the methylation level of POMC in leukocytes obtained from obese men and those who did not gain weight; this study suggested that the methylation status of POMC might function as a useful biomarker for determining a predisposition to obesity. These results, however, should be interpreted carefully, because of the small sample size $(n=18)$. In our study, the sample size was determined based on the standard recommended by Miot (2011), which allowed us to make more confident inferences regarding the analyzed population.

Horvath et al. (2014) revealed a significant positive correlation between whole genome DNA methylation and BMI in liver tissue samples; this suggested that epigenetic factors could be tissue-specific, and highlights the importance of analyzing appropriate tissues to detect the correlations between methylation and BMI.

Indeed, recent studies have supported the theory that the BMI is regulated by a complex circuit involving neuropeptides responsible for maintaining the energy balance and a healthy weight, which are expressed in neurons located at the hypothalamus (Schwartz et al., 2000; Sahu, 2003; Marco et al., 2013; Sobrino Crespo et al., 2014). It must be noted that SNRPN, and some other genes in the 15q11.2-q13 region (such as MAGEL2), are mainly expressed in specific brain regions; this suggested that the modulation of these genes occurs in hypothalamic tissues and not in the blood cells (Bischof et al., 2007; Mercer et al., 2013). This is in agreement with our observation that the MAGEL2 promoter region is highly methylated ( $>90 \%$ ), but barely expressed in lymphocytes obtained from obese and non-obese individuals (results not shown). A comparison of the methylation and age revealed a significant correlation between the levels of methylation in the SNRPN gene and the chronological age, indicating that the methylation was higher in adults than in children. This, in turn, suggested that advancing age increases the level of the SNRPN promoter methylation.

Our results agree with the findings of Fraga et al. (2005) and Day et al. (2013), who also discovered differences in the DNA methylation between young and old monozygotic twin pairs. Obese people have been suggested to suffer from early onset of many age-related pathologies; in addition, SNRPN is known to be related to the onset of obesity (Horvath et al, 2014). However, 
the relationship between increased SNRPN methylation in older subjects and the aging process remains to be further elucidated. The higher level of SNRPN methylation in older individuals (observed in this study) could also reflect the global genomic increase in methylation that occurs naturally with the aging process in an individual (Shen et al., 2005; Kwabi-Addo et al., 2007).

Therefore, we conclude that the DNA methylation profile of the SNRPN promoter region (in blood) is not a useful potential biomarker for the early detection and differential diagnosis of the predisposition to obesity in humans. Although we noticed that older subjects showed a higher degree of SNRPN methylation compared to children, further studies are required to identify any possible correlation between SNRPN expression and the aging process.

\section{Conflicts of interest}

The authors declare no conflict of interest.

\section{ACKNOWLEDGMENTS}

The authors are grateful to the volunteers of the study. Research supported by the Fundação de Amparo a Pesquisa de Minas Gerais/FAPEMIG, Coordenação de Aperfeiçoamento de Pessoal de Nível Superior/CAPES, Conselho Nacional de Desenvolvimento Científico e Tecnológico/CNPq, and the Instituto de Ensino e Pesquisa da Santa Casa de Belo Horizonte/IEP-SCBH.

\section{REFERENCES}

Bischof JM, Stewart CL and Wevrick R (2007). Inactivation of the mouse Mage/2 gene results in growth abnormalities similar to Prader-Willi syndrome. Hum. Mol. Genet. 16: 2713-2719.

Christensen BC, Houseman EA, Marsit CJ, Zheng S, et al. (2009). Aging and environmental exposures alter tissue-specific DNA methylation dependent upon CpG island context. PLoS Genet. 5: e1000602.

Crujeiras AB, Campion J, Diaz-Lagares A, Milagro FI, et al. (2013). Association of weight regain with specific methylation levels in the NPY and POMC promoters in leukocytes of obese men: a translational study. Regul. Pept. 186: 1-6.

Day K, Waite LL, Thalacker-Mercer A, West A, et al. (2013). Differential DNA methylation with age displays both common and dynamic features across human tissues that are influenced by $\mathrm{CpG}$ landscape. Genome Biol. 14: R102.

Fraga MF, Ballestar E, Paz MF, Ropero S, et al. (2005). Epigenetic differences arise during the lifetime of monozygotic twins. Proc. Natl. Acad. Sci. U. S. A. 102: 10604-10609.

Horvath S, Erhart W, Brosch M, Ammerpohl O, et al. (2014). Obesity accelerates epigenetic aging of human liver. Proc. Natl. Acad. Sci. U. S. A. 111: 15538-15543.

Kim SJ, Miller JL, Kuipers PJ, German JR, et al. (2012). Unique and atypical deletions in Prader-Willi syndrome reveal distinct phenotypes. Eur. J. Hum. Genet. 20: 283-290.

Kwabi-Addo B, Chung W, Shen L, Ittmann M, et al. (2007). Age-related DNA methylation changes in normal human prostate tissues. Clin. Cancer Res. 13: 3796-3802.

Marco A, Kisliouk T, Weller A and Meiri N (2013). High fat diet induces hypermethylation of the hypothalamic Pomc promoter and obesity in post-weaning rats. Psychoneuroendocrinology 38: 2844-2853.

Mei Z, Grummer-Strawn LM, Pietrobelli A, Goulding A, et al. (2002). Validity of body mass index compared with other bodycomposition screening indexes for the assessment of body fatness in children and adolescents. Am. J. Clin. Nutr. 75: 978-985.

Mercer RE, Michaelson SD, Chee MJ, Atallah TA, et al. (2013). Magel2 is required for leptin-mediated depolarization of POMC neurons in the hypothalamic arcuate nucleus in mice. PLoS Genet. 9: e1003207.

Miot HA (2011). Tamanho da amostra em estudos clínicos e experimentais. J. Vasc. Bras. 10: 275-278.

Plasschaert RN and Bartolomei MS (2014). Tissue-specific regulation and function of Grb10 during growth and neuronal commitment. Proc. Natl. Acad. Sci. U. S. A. 112: 6841-6847.

Rakyan VK, Down TA, Thorne NP, Flicek P, et al. (2008). An integrated resource for genome-wide identification and analysis of human tissue-specific differentially methylated regions (tDMRs). Genome Res. 18: 1518-1529. 
Reik W and Walter J (2001). Genomic imprinting: parental influence on the genome. Nat. Rev. Genet. 2: 21-32.

Rubatino FVM, Carobin NV, Freitas ML, de Oliveira VT, et al. (2015). Manipulation of primer affinity improves high-resolution melting accuracy for imprinted genes. Genet. Mol. Res. 14: 7864-7872.

Sahu A (2003). Leptin signaling in the hypothalamus: emphasis on energy homeostasis and leptin resistance. Front. Neuroendocrinol. 24: 225-253.

Schilling E and Rehli M (2007). Global, comparative analysis of tissue-specific promoter CpG methylation. Genomics 90: $314-323$.

Schwartz MW, Woods SC, Porte Jr D, Seeley RJ, et al. (2000). Central nervous system control of food intake. Nature 404: 661-671.

Shen L, Kondo Y, Rosner GL, Xiao L, et al. (2005). MGMT promoter methylation and field defect in sporadic colorectal cancer. J. Natl. Cancer Inst. 97: 1330-1338.

Sobrino Crespo C, Perianes Cachero A, Puebla Jimenez L, Barrios V, et al. (2014). Peptides and food intake. Front. Endocrinol. 5: 58.

Souren NY, Tierling S, Fryns JP, Derom C, et al. (2011). DNA methylation variability at growth-related imprints does not contribute to overweight in monozygotic twins discordant for BMI. Obesity 19: 1519-1522.

Suzuki MM and Bird A (2008). DNA methylation landscapes: provocative insights from epigenomics. Nat. Rev. Genet. 9: 465-476.

Wang X, Zhu H, Snieder H, Su S, et al. (2010). Obesity related methylation changes in DNA of peripheral blood leukocytes. BMC Med. 8: 87.

Weinstein LS, Xie T, Qasem A, Wang J, et al. (2010). The role of GNAS and other imprinted genes in the development of obesity. Int. J. Obes. 34: 6-17.

WHO (2000). Obesity: preventing and managing the global epidemic. Report of a WHO consultation. World Health Organ. Tech. Rep. Ser. 894: i-xii, 1-253. 